\title{
Uncertainty Analysis of Measuring Unloaded Quality Factor of a Series Transmission Resonator
}

\author{
A. El-Tager ${ }^{*}$
}

\begin{abstract}
There are different ways to calculate the unloaded bandwidth of a series transmission resonator. For this type of two-port resonators, the availability of both return loss $\left(s_{11}\right)$ and insertion loss $\left(s_{21}\right)$ over-determines the system; that is, the unloaded 3-dB bandwidth and hence the quality factor of the resonator can be calculated from either $s_{21}$ or $s_{11}$. This paper describes different techniques that can be used to determine the unloaded quality factor of a series two-port resonator in order to determine which formulae should be used to most accurately measure the unloaded 3-dB bandwidth of the resonator. Sources of errors are described for both $s_{11}$ and $s_{21}$ methods. Then the measurement uncertainty is analyzed and compared to obtain the most accurate $Q_{0}$ reading.
\end{abstract}

Keywords: resonators, quality factor, measurement uncertainty, microwave circuits.

\section{Introduction}

Microwave resonators are important circuit components for devices like filters, diplexers, multipliers, and oscillators. As such, a fast and accurate characterization of the resonator is essential in assisting circuit designers to accurately predict the performance of any microwave circuit in which the resonator is used. Resonators are characterized by their resonant frequency $\left(f_{0}\right)$ and quality factor $(Q)$. Three important parameters associated with a resonator are the resonator unloaded quality factor $\left(Q_{0}\right)$, the coupling coefficient $(\kappa)$, and the loaded quality factor $\left(Q_{L}\right)$. The unloaded quality factor establishes an upper limit for the resonator performance, while coupling coefficient describes the interaction between the resonator and interfaced microwave circuits. An accurate measurement of the unloaded Q-factor of a microwave resonator is of prime importance. Although the unloaded Q may be calculated theoretically, it cannot be directly measured in practice. Therefore, It was an attractive area of research starting from slotted line measurements [1] and ending with vector network analyzers (VNA) [2,3]. Q-factor measurements could be either reflection type or transmission type[4,5]. The latter is very popular $[6,7,8]$ and is investigated in this paper.

This paper describes different techniques that can be used to determine the unloaded quality factor of a series transmission resonator. One of these techniques is using transmission parameter $s_{21}$ and the loaded 3-dB points. The uncertainty of this technique is analyzed in [5].

\footnotetext{
*Egyptian Armed Forces, aeltager@yahoo.ca
} 
In this paper two other techniques; namely using $s_{21}$ and the unloaded 3-dB points, and using return loss $s_{11}$ and the unloaded 3-dB points, are discussed and analyzed. The availability of both $s_{11}$ and $s_{21}$ over-determines the system; that is, $s_{21}$ can be calculated based on the knowledge of $s_{11}$ and vice versa. This paper determines which formulae should be used to most accurately measure the unloaded 3-dB bandwidth of the resonator. Sources of errors are described for both $s_{11}$ and $s_{21}$ methods. Then the measurement uncertainty is analyzed and compared to obtain the most accurate $\mathrm{Q}_{0}$ reading.

\section{Methods of Measuring the Unloaded Quality Factor}

This section describes the different techniques that can be used to determine the unloaded quality factor of a series two-port (transmission) resonator. The equations presented here are based on those which have been derived in [5].

\section{a) Using the Loaded 3-dB Points and $s_{21}$}

This technique is the most often encountered in practice. It is found that the loaded and unloaded Qs are related through Equation 1 [2,5].

$Q_{0}=(1+2 \kappa) Q_{L}$

It is also seen that the $s_{21}$ parameter at resonance, which is real valued, is given by Equation 2 [5].

$s_{21}^{r e s}=\frac{2 \kappa}{1+2 \kappa}$

By isolating $\kappa$ from Equation 2 and substituting the value into Equation 1, the result of Equation 3 is obtained.

$Q_{0}=\frac{Q_{L}}{1-s_{21}^{r e s}}$

The $s_{21}$ parameter at resonance is easily obtained, leaving $Q_{L}$ to be measured if Equation 3 is to be used. Also, the squared magnitude of $s_{21}$ at the loaded 3-dB points can be obtained:

$\left|s_{21 L}\right|^{2}=\frac{2 \kappa^{2}}{(1+2 \kappa)^{2}}$

Close inspection of Equations 2 and 4 reveals that the squared magnitude of $s_{21}$ at the loaded $3 \mathrm{~dB}$ points is exactly half the squared magnitude of $s_{21}$ at resonance $\left(s_{21}{ }^{\text {res }}\right)$. This means that the loaded $3 \mathrm{~dB}$ points occur at $s_{21}{ }^{\text {res }}-3 \mathrm{~dB}$ exactly, which provides a quick method of determining $\mathrm{Q}_{\mathrm{L}}$ for use in Equation 3.

\section{b) Using the Unloaded 3-dB Points and $s_{21}$}


Equation 2 allows the coupling coefficient $\kappa$, which is frequency invariant, to be evaluated at resonance. Equation 5, provides the unloaded 3-dB points of $\mathrm{s}_{21}$ directly, allowing $\mathrm{Q}_{0}$ to be calculated directly.

$$
\left|s_{21 U}\right|^{2}=\frac{2 \kappa^{2}}{1+2 \kappa+2 \kappa^{2}}
$$

\section{c) Using the Unloaded 3-dB Points and $s_{11}$}

If one has access to the reflection coefficient magnitude, then the coupling coefficient may be extracted using Equation 6.

$$
\Gamma_{\text {res }}=\frac{1}{1+2 \kappa}
$$

The unloaded 3-dB points are then given by Equation 7, again allowing $\mathrm{Q}_{0}$ to be measured directly. There is no simple "-3 dB expression" for the reflection coefficient similar to that found for the $s_{21}$ parameter of Method (a).

$$
\left|\Gamma_{U}\right|^{2}=\frac{1+2 \kappa+2 \kappa^{2}}{(1+2 \kappa)^{2}}
$$

\section{Description of the Sources of Errors}

In a perfect world, each of the three methods presented above would yield the same value for $Q_{0}$. However, uncertainty is present in all practical measurement systems, and the effects of uncertainty on our desired value must be determined. The effects of system uncertainty on the results of Method (a) have been discussed in a recent paper by Kajfez [5]. In this paper, the uncertainty analyses for Methods (b) and (c) are derived in sections 4 and 5 respectively. It is assumed that the network analyzer has an absolute amplitude level uncertainty of $\Delta \mathrm{p} d \mathrm{~B}$ on the $\mathrm{s}_{21}$ reading and $\Delta \mathrm{rdB}$ on the $\mathrm{s}_{11}$ reading. Additionally, it is assumed that there exists a frequency uncertainty $\Delta \mathrm{f}$ when reading the bandwidth. The resonant frequency uncertainty is assumed to be error-free.

\section{Uncertainty Analysis for Method (b), Using $s_{21}$ to Find the Unloaded 3-dB Points}

The first source of uncertainty using this method is in determining $\kappa$ using Equation 2 because there is a level uncertainty in the $s_{21}$ parameter at resonance. If p denotes the $s_{21}$ magnitude at resonance in decibels (a negative value), with an uncertainty of $\Delta \mathrm{p} d B$, then $\kappa$ can be expressed as Equation 8.

$$
\kappa=\frac{10^{p / 20}}{2\left(1-10^{p / 20}\right)}
$$


It is a straightforward task to prove that the absolute (linear) uncertainty of $\kappa$ is given by Equation 9.

$$
\Delta \kappa=\kappa \frac{\ln 10}{20\left(1-10^{p / 20}\right)} \Delta p
$$

Equation 5 is then used to calculate the unloaded $3 \mathrm{~dB}$ level of $\mathrm{s}_{21}$. This calculated level has an uncertainty due to the uncertainty of $\kappa$. If the $\kappa$ uncertainty is propagated in Equation 5 , the resulting uncertainty of the calculated $\mathrm{s}_{21}$ level, expressed in decibels, is obtained as per Equation 10.

$$
\Delta s_{21 U}^{d B}(\kappa)=\frac{20}{\ln 10} \cdot \frac{1+1 / \kappa}{1+2 \kappa+2 \kappa^{2}} \Delta \kappa
$$

When Equation 5 is used to find the unloaded 3-dB level, designers are again faced with the uncertainty of the VNA of $\Delta \mathrm{p} \mathrm{dB}$ when they try to establish this level. This additional uncertainty at the $3 \mathrm{~dB}$ point is assumed to be independent of the uncertainty that was present at resonance, so the overall uncertainty of the unloaded 3-dB level, expressed in decibels, becomes that of Equation 11.

$$
\Delta s_{21 U}^{d B}=\sqrt{[\Delta p]^{2}+\left[\Delta s_{21 U}^{d B}(\kappa)\right]^{2}}
$$

Later, linear value of $\left|s_{21}\right|^{2}$ is used, and its (linear) error is given by Equation 12 by means of Equation 11. So how can Equation 12 be used to find the uncertainty of the measured bandwidth?

$$
\Delta s_{21 U}^{2}=\left|s_{21 U}\right|^{2} \frac{\ln 10}{10} \Delta s_{21 U}^{d B}
$$

The missing step is now provided. From Fig. 1 it can be seen that the $s_{21}$ parameter can be formulated as Equation 13.

$$
s_{21}=\frac{2 Z_{0}}{Z_{L}+2 Z_{0}}
$$

Where the unloaded series transmission resonator is expressed as $Z_{L}=R+j X$ as shown in Fig.1. Thus, the squared magnitude of $s_{21}$ near resonance can be expressed as Equation 14, if using $\kappa=Z_{0} / R$ and $Z_{L} \approx R\left(1+j Q_{0} B W\right)$ near resonance, where $B W$ is the double-sided fractional bandwidth.

$$
\left|S_{21}\right|_{f \approx f_{0}}^{2}=\frac{4 \kappa^{2}}{(2 \kappa+1)^{2}+Q_{0}^{2} B W^{2}}
$$




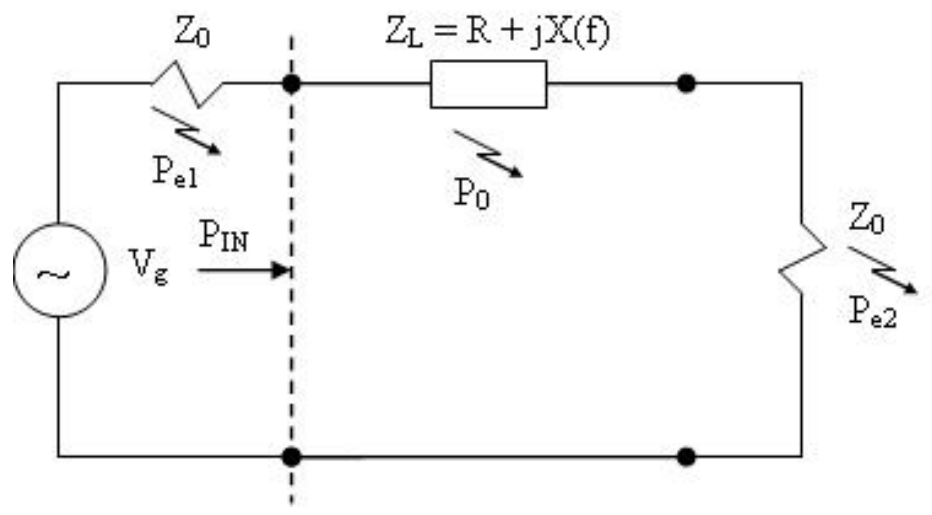

Fig. 1. Two port circuit model for a series transmission resonator

Now, its is time to introduce uncertainties into Equation 14. The first uncertainty is that of $s_{21}$ given by Equation 12. The coupling coefficient has no uncertainty here because Equation 14 has been derived analytically. Hence BW has an absolute uncertainty that is expressed by $\triangle B W\left(s_{21}\right)$. Using the fact that $Q_{0} B W=1$ at resonance, propagating the uncertainty through Equation 14 yields Equation 15.

$\Delta B W\left(s_{21}\right)=\frac{\left(1+2 \kappa+2 \kappa^{2}\right)^{2}}{2 \kappa^{2} Q_{0}} \Delta s_{21 U}^{2}$

The VNA is also assumed to have a bandwidth uncertainty of $\Delta f$, so the total uncertainty of the bandwidth is given by Equation 16 .

$\Delta B W=\sqrt{\left[\frac{\Delta f}{f_{0}}\right]^{2}+\left[\Delta B W\left(s_{21}\right)\right]^{2}}$

Since $Q_{0}=1 / B \mathrm{~W}$, the absolute and relative uncertainties of $Q_{0}$ are given by Equations 17 and 18 , respectively, completing the derivation.

$$
\begin{aligned}
& \Delta Q_{0}=\frac{\Delta B W}{B W^{2}} \\
& \frac{\Delta Q_{0}}{Q_{0}}=\frac{\Delta B W}{B W}
\end{aligned}
$$

\section{Uncertainty Analysis for Method (c), Using $s_{11}$ to Find the Unloaded 3-dB Points}

If Equation 7 is used to calculate the unloaded $3 \mathrm{~dB}$ points, a different $Q_{0}$ uncertainty will result. From Equation 6, knowing that $\mid \Pi$ has an uncertainty of $\Delta r$, the absolute linear uncertainty of the coupling coefficient becomes that of Equation 19. 


$$
\Delta \kappa=(1+2 \kappa) \frac{\ln 10}{40} \Delta r
$$

Propagating this error through Equation 7, the absolute uncertainty of the 3-dB $\mathrm{s}_{11}$ level, expressed in decibels, is given by Equation 20.

$$
\Delta s_{11 U}^{d B}(\kappa)=\frac{20}{\ln 10} \cdot \frac{1+2 \kappa}{1+2 \kappa+2 \kappa^{2}} \Delta \kappa
$$

The reading of this $s_{11}$ level is subjected to the analyzer's uncertainty of $\Delta r$, and if this uncertainty is uncorrelated with the uncertainty at resonance, the total uncertainty of the 3-dB $\mathrm{s}_{11}$ level is given in decibels by Equation 21, and the absolute linear error of $\left|\mathrm{s}_{11 U}\right|^{2}$ is given by Equation 22.

$$
\begin{aligned}
& \Delta s_{11 U}^{d B}=\sqrt{[\Delta r]^{2}+\left[\Delta s_{11 U}^{d B}(\kappa)\right]^{2}} \\
& \Delta s_{11 U}^{2}=\left|s_{11 U}\right|^{2} \frac{\ln 10}{10} \Delta s_{11 U}^{d B}
\end{aligned}
$$

The reflection coefficient can be expressed as Equation 23, from which its squared magnitude near resonance is approximated by Equation 24.

$$
\begin{aligned}
& s_{11}=\frac{Z_{L}}{Z_{L}+2 Z_{0}} \\
& \left|s_{11}\right|_{f \approx f_{0}}^{2}=\frac{1+Q_{0}^{2} B W^{2}}{(2 \kappa+1)^{2}+Q_{0}^{2} B W^{2}}
\end{aligned}
$$

Propagating the uncertainty of $\left|s_{11}\right|^{2}$ through Equation 24, one obtains the uncertainty of the bandwidth due to that of $s_{11}$, and this is given by Equation 25 .

$$
\Delta B W\left(s_{11}\right)=\frac{\left[(2 \kappa+1)^{2}+1\right]^{2}}{2 Q_{0}\left[(2 \kappa+1)^{2}-1\right]} \Delta s_{11 U}^{2}
$$

The total bandwidth uncertainty and the resulting $Q_{0}$ uncertainties are again given by Equations 16 through 18.

\section{Analysis and Comparison of the Uncertainties}

The uncertainties of the three above methods are more investigated and compared in this section. Using only $s_{21}$, we first compare the relative uncertainties of $Q_{0}$ for Methods (a) and (b) for the case of $\Delta \mathrm{p}=0.1 \mathrm{~dB}, \Delta \mathrm{f}=1 \mathrm{kHz}$, and $Q_{0}=10000$. The results are shown in Fig. 2.

The results of Fig. 2 indicate that Method (a) always yields a smaller uncertainty. This shouldn't be that surprising, since Method (a) does not use a calculation to determine the 3-dB points; instead, the level is $3 \mathrm{~dB}$ below resonance, exactly. Method (b) uses a function of $\kappa$ to 
determine the unloaded 3-dB points, a calculation that amplifies the uncertainty. So the uncertainty of the coupling coefficient causes Method (b) to have more uncertainty than that of Method (a).

Figure 2 also shows that the accuracy of both methods degrades as the coupling coefficient increases. Upon careful reflection, this is due to the fact that the magnitude of $s_{21}$ at resonance increases with the coupling coefficient. If the level error of $\Delta \mathrm{p} d \mathrm{~B}$ is constant, this means that as $s_{21}$ increases, its absolute uncertainty also increases. As an example, $\pm 0.1 \mathrm{~dB}$ for a level of $-30 \mathrm{~dB}$ represents a much smaller absolute uncertainty than does, say, $\pm 0.1 \mathrm{~dB}$ for a level of $-5 \mathrm{~dB}$. Hence the increase in uncertainty with $\kappa$ is due directly to the fact that $\Delta \mathrm{p}$ is constant.

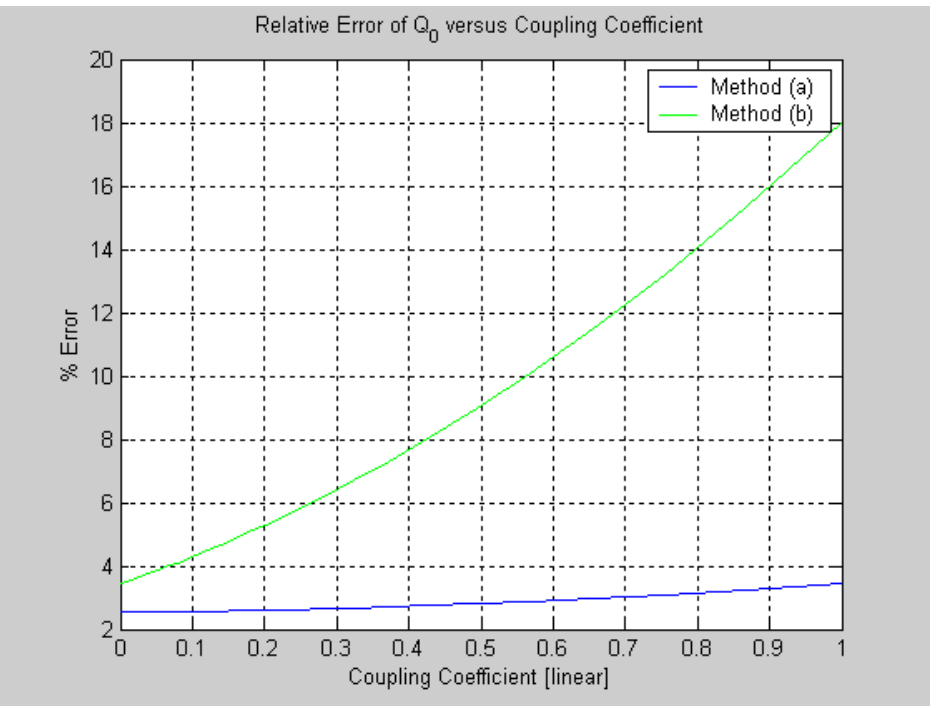

(a)

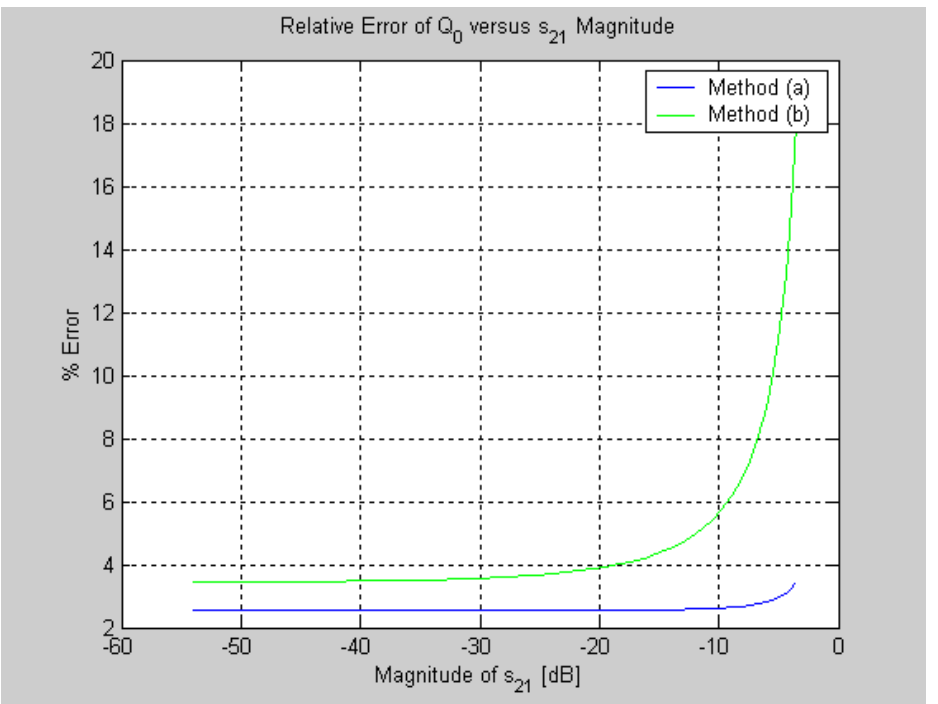

(b)

Fig. 2. Relative $Q_{0}$ Uncertainty versus Coupling Coefficient and Magnitude of $S_{21}$ for $\Delta p=0.1 \mathrm{~dB}, \Delta f=1 \mathrm{kHz}$, and $Q_{0}=10000$ Using Methods (a) and (b) 
Given this fact, the reflection coefficient at resonance decreases as the coupling coefficient is increased. Hence the absolute uncertainty of $s_{11}$ should go down as $\kappa$ is increased. Method (c) is expected to be more accurate above a certain coupling coefficient level. Comparisons of the relative $Q_{0}$ uncertainty using Methods (a) and (c) are shown in Fig. 3 using the same parameters as those of Fig. 2.

The results using Method (a) are identical to those published by Kafjez [5]. Using our uncertainty assumptions, and the assumption that $\Delta \mathrm{p}=\Delta \mathrm{r}$, it is clear that $\mathrm{s}_{11}$ should be used instead of $\mathrm{s}_{21}$ above a certain coupling level. This crossover level is given by $\kappa_{\text {crossover }} \approx 1.255$ or $\left|\mathrm{s}_{21}{ }^{\text {res }}\right|_{\text {crossover }} \approx-2.915 \mathrm{~dB}$. These levels are irrespective of the $\Delta \mathrm{p}, \Delta \mathrm{f}$, and $\mathrm{Q}_{0}$ levels, although the resulting maximum relative uncertainty of $\mathrm{Q}_{0}$ at the crossover point is of course a function of all of these parameters.

Taking the maximum $\mathrm{Q}_{0}$ uncertainty as corresponding to that at the $\kappa_{\text {crossover }}$ level, we can plot the contributions to $\Delta \mathrm{Q}_{0}$ separately. Equation 16 indicates that the absolute uncertainty of $\mathrm{Q}_{0}$ is due to two contributions: one due to $\Delta \mathrm{f}$ and the other due (indirectly) to $\Delta \mathrm{r}$ or $\Delta \mathrm{p}$. Combining Equations 16 and 18, the relative uncertainty of $\mathrm{Q}_{0}$ can be expressed as Equation 26. If $\Delta \mathrm{f}$ is zero, the relative uncertainty of $\mathrm{Q}_{0}$ due to the level uncertainty $\Delta \mathrm{p}=\Delta \mathrm{r}$ will be given by Equation 25 without the $\mathrm{Q}_{0}$ in the denominator, that is, this contribution to the relative $\mathrm{Q}_{0}$ uncertainty will not be a function of $\mathrm{Q}_{0}$.

$$
\frac{\Delta Q_{0}}{Q_{0}}=\sqrt{\left[\frac{\Delta f Q_{0}}{f_{0}}\right]^{2}+\left[Q_{0} \Delta B W\left(s_{11}\right)\right]^{2}}
$$

The maximum contribution due to $\Delta \mathrm{p}=\Delta \mathrm{r}$ to the relative uncertainty of $Q_{0}$, that is, $Q_{0} \Delta B W\left(s_{11}\right)$, is plotted in Fig. 4. for $\kappa=1.255$. The other term of Equation 26 depends on the parameters of the resonator and can be easily evaluated.

\section{Conclusion}

This paper describes three main techniques that can be used to determine the unloaded quality factor of a series transmission resonator. First, using transmission parameter $S_{21}$ and the loaded 3-dB points. The uncertainty of this technique is analyzed in [5]. Second, using $S_{21}$ and the loaded 3-dB points. And third, using return loss $S_{11}$ and the unloaded 3-dB points. Second and third techniques are discussed and analyzed. This paper determines which technique should be used to most accurately measure the unloaded 3-dB bandwidth of the resonator. Sources of errors are described for both $s_{11}$ and $s_{21}$ methods. Then the measurement uncertainty is analyzed and compared to the first technique (method a) to obtain the most accurate $Q_{0}$ reading. For the second technique (method b) it is worse than the first which is matched with theory. For the third technique (method c), it is found better than method (a) for coupling factors larger than certain value ( $\kappa_{\text {crossover }} \approx 1.255$ ), otherwise, method (a) is better. Therefore, given the uncertainties in the measurement system, one can determine which parameter $\left(s_{11}\right.$ or $\left.s_{21}\right)$ to use to obtain the most accurate $Q_{0}$ reading. Furthermore, the maximum uncertainty is calculated, which occurs for the special case of $\kappa=1.255$. 


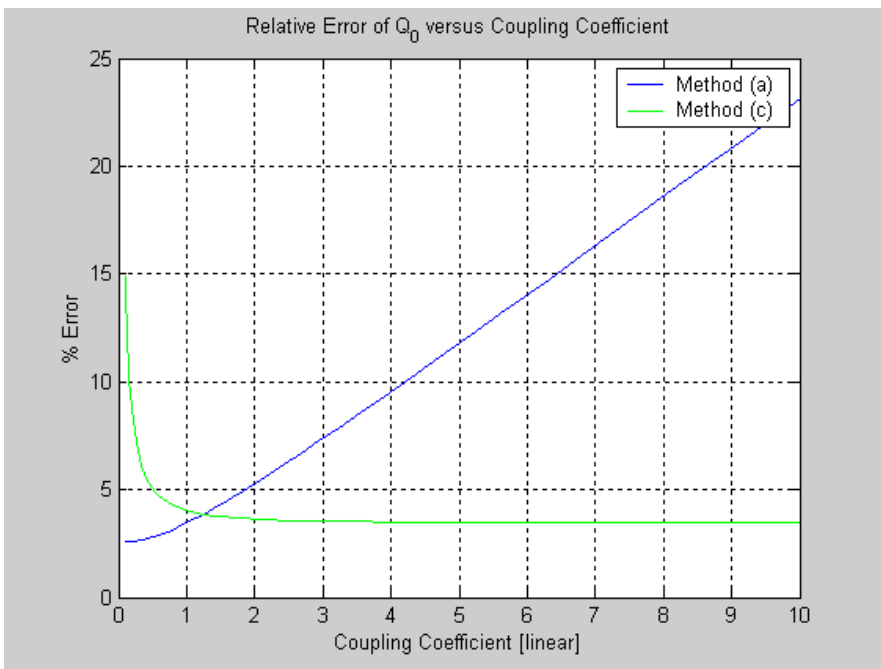

(a)

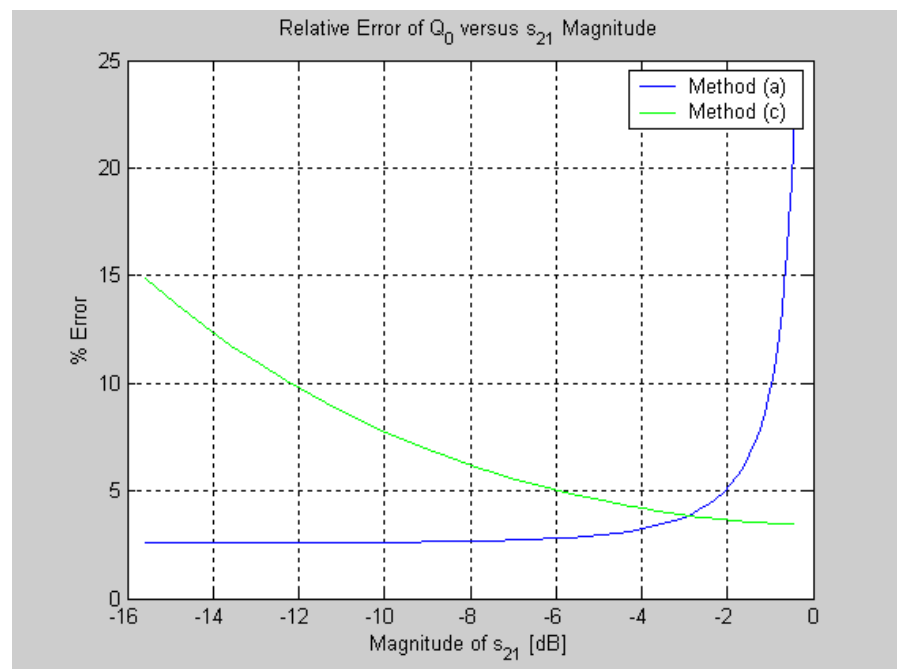

(b)

Fig. 3. Relative $Q_{0}$ Uncertainty versus Coupling Coefficient and Magnitude of $S_{21}$ for $\Delta r=\Delta p=0.1 \mathrm{~dB}, \Delta \mathrm{f}=1 \mathrm{kHz}$, and $\mathrm{Q}_{0}=10000$ Using Methods (a) and (c) 


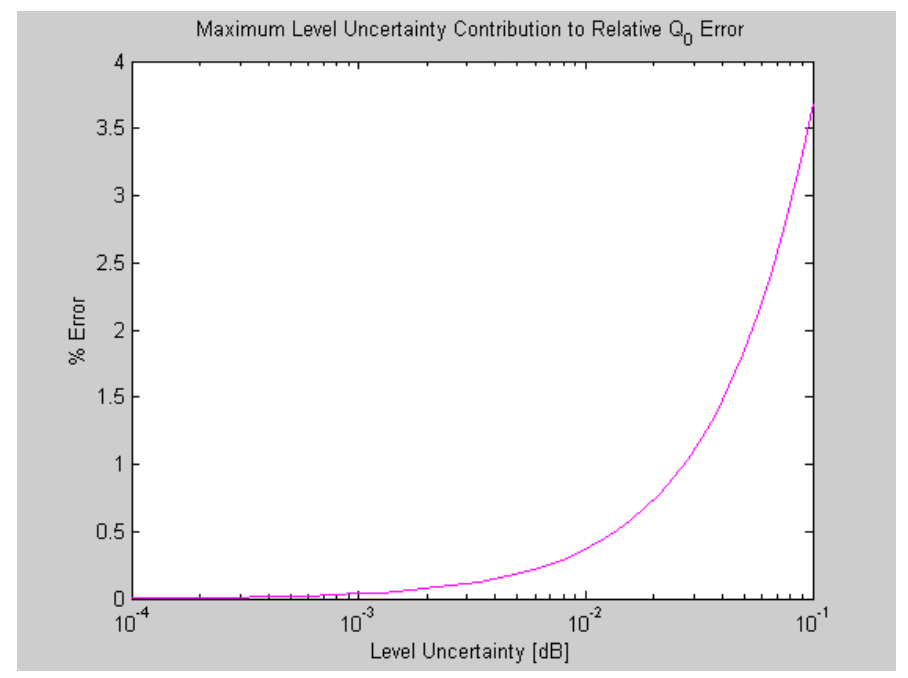

Fig. 4. Maximum Error Contribution of the Level Uncertainty to the Relative Uncertainty of $\mathbf{Q}_{0}$

\section{References}

[1] E. L. Ginzton, , Microwave Measurements, New York, McGraw-Hill, 1957.

[2] D. Kajfez and E.J. Hwan, "Q-Factor measurement with network analyzer", IEEE Trans. Microwave Theory Tech., Vol. MTT-32, July 1984, pp.666-670.

[3] R. S. Kwok and J. Liang, " Charecterization of High Q-Factor Resonators for Microwave-filter Applications", IEEE Trans. Microwave Theory Tech., Vol. MTT-47, No.1, Jan. 1999, pp.111-114.

[4] L. Chua and D. Mirshekar-Syahkal, " Accurate and Direct Characterization of High-Q Microwave Resonators Using One-Port Measurement", IEEE Trans. Microwave Theory Tech., Vol. MTT-51, No.3, March 2003, pp.978-985.

[5] D. Kajfez et al., "Uncertainty Analysis of the Transmission-Type Measurement of QFactor", IEEE Trans. Microwave Theory Tech., 47, March 1999, pp.367-371.

[6] R. K. Mongia and R. K. Arora, "Equivalent Circuit Parameters of an Aperture coupled Open Resonator Cavity", IEEE Trans. Microwave Theory Tech., MTT-41, Aug. 1993, pp.1245-1250.

[7] D. A. Rudy and J. P. Mendelson, " Measurement of RF dielectric Properties with Series Resonant Microstrip Elements", Microwave J., Vol. 41, March 1998, pp.22-41.

[8] J. Sheen, " Measurements of microwave dielectric properties by an amended cavity perturbation technique ", Science Direct Measurement J., Volume 42, Issue 1, January 2009, Pages 57-61. 